Research Article

\title{
PVDF Piezoelectric Sensors for Seeds Counting and Coulter Clogging Detection in Sowing Process Monitoring
}

\author{
Lukasz Gierz $\mathbb{D}^{1}$ and Bartłomiej Kazimierz Paszkiewicz $\mathbb{D}^{2}$ \\ ${ }^{1}$ Poznan University of Technology, Faculty of Civil and Transport Engineering, Piotrowo 3, 60-965 Poznan, Poland \\ ${ }^{2}$ Wroclaw University of Science and Technology, Faculty of Microsystem Electronics and Photonics, Wrocław, Poland \\ Correspondence should be addressed to Bartłomiej Kazimierz Paszkiewicz; bartlomiej.paszkiewicz@pwr.edu.pl
}

Received 6 November 2019; Revised 26 January 2020; Accepted 9 April 2020; Published 20 May 2020

Academic Editor: Jiun-Wei Horng

Copyright (C) 2020 Łukasz Gierz and Bartłomiej Kazimierz Paszkiewicz. This is an open access article distributed under the Creative Commons Attribution License, which permits unrestricted use, distribution, and reproduction in any medium, provided the original work is properly cited.

\begin{abstract}
The paper presents the results of a test on the use of piezoelectric sensors made of PVDF foil for counting grain and checking clogging in the sowing drill. The tests were carried out in a simulator of the sowing drill with the use of a measuring system with high input impedance. Two kinds of barley and triticale grains were investigated. Measurements were carried out for different feeding frequencies. On the basis of the signals recorded, an algorithm of counting the sown grain was developed and tested. The algorithm is based on signal statistic comparison in adjacent windows. The developed algorithm allows counting more than $90 \%$ of the sown grains.
\end{abstract}

\section{Introduction}

In modern agriculture industry, the two key issues determining the economic efficiency of arable land and industrial equipment utilization is the challenge of obtaining a uniform grain distribution over the entire field and the detection of seeder coulter clogging [1-6]. Solving both issues allow increasing the efficiency of food production while reducing the use of herbicides, fertilizers, and water. The first issue, seeding uniformity, allows for more precise cultivation. This is due to the fact that it is possible to predict the optimum demand for fertilizers, herbicides, and water. If the seeding is not evenly distributed, the application of different agents on a whole area will result in local deficits or excesses. Similarly, the second challenge, avoiding clogging, is important in terms of production efficiency. Clogging of one of the seed drill coulters results in the presence of areas where no seed has been sown and a decrease in overall production capacity per hectare. The basic parameter used in the process is the sowing frequency, a measure that describes the amount of seeds per area. Usually, it is determined by the number of seeds per square meter $[7,8]$. Optical measurement methods are used in classical solutions for the determination of sowing frequency [9]. However, they are unreliable due to the high dustiness during the sowing process. A promising alternative is the use of polymer or ceramics piezoelectric sensors to detect sown seeds. Due to the low price of such sensors, their high strength, high operating frequencies, and high signal-to-noise ratio, they allow for the accurate counting of the sown seeds $[10,11]$. The existing literature on the use of piezoelectric sensors is limited. The work by Huang et al. [12] describes a system using PVDF film in the form of a cantilever beam that uses the voltage generated by the subsequent incident seeds to monitor the seeding process. When voltage pulses are not generated, it suggests that clogging has occurred in the system. In the paper [13], the seeding and fertilizing system is described. The fertilizer control module uses PVDF film to detect stress in the supply pipe that is created as a result of clogging. In that case, a piezoelectric sensor is used as a stress measuring device. In addition, there are commercial systems that apply piezoelectric sensors; e.g., AIRidium ${ }^{\circledR}$ System from MüllerElektronik is able to count the incident grain with a frequency of up to $10 \mathrm{kHz}$ [14]. However, the manufacturer does not provide technical data on the type of materials used, signal processing algorithms, and real counting efficiency. 
The principle of piezoelectric sown seed counting systems is the utilization of thin piezoelectric material with electrodes on both sides. The foil is placed in the seeder system in a way that the pneumatically driven seeds collide with it on the way between the seed tank and the coulter. This causes piezoelectric polarization of the material that can be observed as voltage changes or current flows between the sensors electrodes. The key factor for proper operation is an optimal design of the sensor and the development of an algorithm for signal processing to acquire complete information about the collisions. The first step in the development process is to obtain the time and frequency characteristics of electrical signals generated by collisions of different types of seeds with piezoelectric foil. The article presents the results of measurements of the electrical response of the custom designed piezoelectric sensor to be applied for counting of the number of seeds sown by the multicoulter seed drill. Measurements are carried out using a laboratory setup that simulates the work of the seed drill. The analyzed signals come from single hits as well as from hit series. An efficient signal processing algorithm has been proposed for counting the number of impacts and the efficiency of the algorithm is evaluated.

\section{Materials and Methods}

2.1. Sowing Process Experimental Setup. In order to analyze the phenomenon of collisions with piezoelectric foil, an experimental installation was set up. A photo of it is presented in Figure 1.

Pneumatically driven seeds in the seed drill reach a certain speed depending on their weight, size, and aerodynamic shape. The blower fan speed has been selected to be two times larger than in regular drill in order to enhance examined phenomena $[15,16] ;[17-19]$. The measured air velocity was about $50 \mathrm{~ms}^{-1}$, and the diameter pipe was $28 \mathrm{~mm}$. Seeds velocities, measured using high speed camera, were in the range of $8-12 \mathrm{~ms}^{-1}$, and measured cx coefficients of seeds were $0.37 \pm 0.14$ for Barley winter (Titus) and $0,4 \pm 0,004$ for Triticale (Borwo) [20]. The idea behind the developed measuring system is to place a piezoelectric sensor in such a way that the seeds hit it, thus generating a voltage signal. Collisions time is in a range of single milliseconds [21]. In order to determine the correct configuration of the measuring system, it is necessary to determine the characteristics of these collisions and to determine the natural frequencies of the piezoelectric foil and measuring system. The developed sensor construction consists of several layers connected by a flexible band. In Figure 2, the cross section of the sensor is presented:.

The grains hit the $1 \mathrm{~mm}$ thick front layer of rubber, which is used to absorb the impact energy of the grain and to extend the interaction time. It also reduces the effect of grains rotation and different impact angles. The energy transferred to the thin rubber layer deforms the piezoelectric film and induces voltage signal. Aluminum plates connected with springs with a thickness of $5 \mathrm{~mm}$ and $2 \mathrm{~mm}$, respectively, serve as an insulator of external vibrations and a mounting bracket. Different compositions of plate

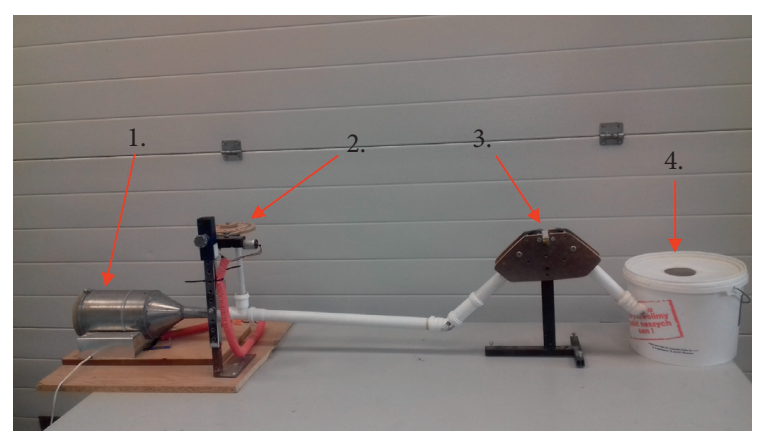

Figure 1: Mechanical design of experimental rig (1-blower, 2-seeds feeding, 3-piezoelectric film foil, 4-tank).

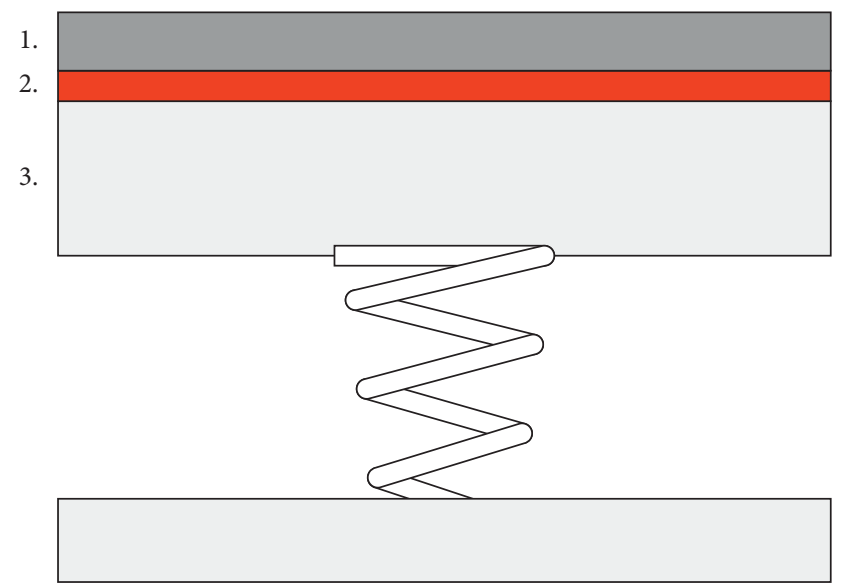

FIgURE 2: Sensor cross section (1-rubber layer, 2-PVDF film, 3-alumina plates with spring amortization).

thicknesses from $1 \mathrm{~mm}$ to $5 \mathrm{~mm}$ were analyzed. Oscilloscopic observations suggested that with such a combination for the established seeding parameters, the signal quality was the best. The front surface of the sensor can be adjusted freely depending on the size of grain delivery tubes; in the experimental setup it was $1 \mathrm{~cm}$ by $3 \mathrm{~cm}$. Approximately this size will be required for application in seed drills that are planned to be first customized into practical use: AKPIL (Drill 500, Tiger, Gepard, Tornado) and Farnet (Falcon and Monsun Families). In the proposed sensors, a foil made of PVDF (Poly (vinylidene fluoride)) was used that is typically applied for the fabrication of piezoelectric sensory elements. It was selected because of several advantages over the other materials $[10,22]$. PVDF foil has much higher elasticity than other crystalline piezoelectric materials applied in the form of the micrometer thin layers such as PZT ceramics (lead, titanium, and zirconium oxide alloys), AlN (AluminumNitride) layers or BaTiO3. Due to its widespread use in conditions of high vibrations and strong environmental hazards, PVDF film is expected to last longer. Additionally, due to the expected industrial scale production of the proposed solution, an important factor is its low cost. In the case of PVDF films, it came from the simplicity of the sensor fabrication process. In contrast to AlN, PVDF foil in sensor applications is usually placed in a layered system. It is coated on both sides with a layer of metallic electrodes made with 
silver-doped ink and a protective layer made of resistant polymer, e.g., Mylar (ethylene terephthalate). The most important parameters important for the use of foil for seeds impact detection are presented in Table 1.

The electric equivalent model of the foil is shown in Figure 3 together with the measuring system, which uses a measuring card with high impedance analog inputs (>10 G $\Omega$ $\| 100 \mathrm{pF}$ ) and high ADC dynamics (16 bits).

Piezoelectric foil can be analyzed electrically as a voltage source connected in series with a certain capacity $C_{0}$, which depends on the foil thickness (inverse dependence) and its surface (proportional dependence). The voltage generated by a voltage source is proportional to the force exerted on the foil, causing the generation of the charge between its electrodes. The voltage value at the terminals is strongly influenced by the measuring instrument connected to them [23]. Due to the finite value of the input impedance, a voltage divider is created between the capacitor $C_{0}$ and the input impedance of the $R_{\mathrm{DAQ}}$ device that causes the change of the measured signal value depending on the frequency, creating a high-pass filter with a time constant $t$ equal to

$$
\tau=2 \pi R_{\mathrm{DAQ}} C_{0} .
$$

In the examined configuration, the DAQ (Data Acquisition) NI myDAQ card was used for dynamic signal acquisition. The input impedance value in this model is above $10 \mathrm{G} \Omega$ that results in a high value of the time constant and the possibility of generating voltages exceeding the measurement range, thus creating the risk of damage to the ADC of the measurement card. For this purpose, a $10 \mathrm{k} \Omega-100 \mathrm{k} \Omega$ resistor has been connected in parallel in the measuring circuit in order to reduce the time constant and to limit the output voltage. In such a circuit, the voltage value that is measured and analyzed is a derivative of the seed contact force during a collision. With this setup, it is possible to precisely observe the course of the collision and the process of energy and momentum transfer to the piezoelectric foil.

\section{Results and Discussion}

3.1. Single Hits. The seeds impacts were recorded in two basic modes. Initially, the seeds were fed by means of a rotary feeder, which ensured that the seeds were fed to the ejection mechanism at equal intervals. The rotary feeder was made in the form of two wheels placed on top of each other. The lower one is stationary, and the upper one is driven by a gear motor $(10 \mathrm{sec} / \mathrm{rpm})$. There are 23 holes drilled on the top wheel and only one, larger, on the bottom wheel. Holes in both wheels match each other positions. Grains are placed in the upper holes when the holes overlap during rotation, the grain is gravitationally fed into the experimental rig. One to three seeds were dosed each time. Barley and triticale seeds were thrown in. The analog signal was recorded by a data acquisition card connected to a PC. The data was recorded in the form of a voltage signal for later analysis with frequencies of $50 \mathrm{k}-100 \mathrm{k}$ samples per second. Figures $4-7$ show selected recoded impacts for all types of tested seeds.

It was observed that despite the different weight and shape of the grains which resulted in their different flight
TABLE 1: PVDF foils parameters important for the proposed application.

\begin{tabular}{lcc}
\hline Parameter & Value & Unit \\
\hline Piezoelectric coefficient $\mathrm{d}_{31}$ & 23 & $10^{-12} \mathrm{CN}^{-1}$ \\
Piezoelectric coefficient $\mathrm{d}_{33}$ & -33 & $10^{-12} \mathrm{CN}^{-1}$ \\
Capacitance $C_{\mathrm{u}}$ & 380 & $\mathrm{pFcm}^{-2}, 1 \mathrm{kHz}, 28 \mu \mathrm{m}$ \\
Young modulus $E$ & $2-4$ & $\mathrm{GPa}$ \\
Dielectric permittivity & $12-13$ & - \\
Contact resistance & 0.1 & $\mathrm{ohm} / \square$ \\
\hline
\end{tabular}

speed and gained kinetic energy, it is not possible to recognize the type of seed on the basis of a recorded electrical signal.

Due to the complex shape of the feeder cables and the sensor area, the signals recorded from single hits may vary considerably. In Figures 4 and 7, one can see barley and triticale impacts, which have made the sensor area resonate. The resonance frequency is $4000-4500 \mathrm{~Hz}$, and the amplitude loss factor $\alpha=0.5$. However, the loss factor is not always constant throughout the relaxation period. A different response can be observed in the second half of the first period. In the case of the impact shown in Figure 4, the reduction of the initial signal can be observed much faster, with the same total relaxation time compared to Figure 7 .

The analysis of Figures 5 and 6 shows the impacts of a completely different course (presumably the grains hit under a different angle). The attenuation coefficient is $\alpha=1$ and the main frequency is $1600-1800 \mathrm{~Hz}$. The impact induced a completely different mode of sensor resonation. Additionally, different sensor responses can be observed after the first period. In the case of Figure 5, a continuous relaxation process can be observed, and in the case of Figure 6 , the signal is clearly dimmed by non-Gaussian noise.

However, the impact energy was sufficient to measure the response of the piezoelectric sensor, connected into the described system, and to count the number of seeds. Additionally, a high variation of maximum signal amplitude was observed. It results from the position of the sensor in the system and the variable amount of energy transmitted during each impact. Although the seeds are of standardised size and mass, and therefore a uniform airflow will cause similar speeds to be achieved, the angle of impact of the seeds is variable due to the shape of the supply pipes, which results in the signal amplitude variation. In addition, the shape of the signal is influenced by additional artifacts when the seeds reflected from the walls of the supply cable strike the sensor again. As can be seen in the figures above, the grains hitting generate very different signals. They can be described by sinusoidal waveforms with a variable attenuation coefficient $\beta$. On the basis of the analysis of the measured signals, it was estimated that the basic frequency of the sensor's response to impact was in the range of $1-2 \mathrm{kHz}$. This is due to the duration of the impact, Eigenfrequencies of the sensor and the measuring system circuit design. Under real-life conditions, the seed for a single coulter is fed at a rate of $20-250 \mathrm{~Hz}$. This information, together with the measured single hits, enables the development of an effective impact counting and nozzle clogging detection algorithm. 


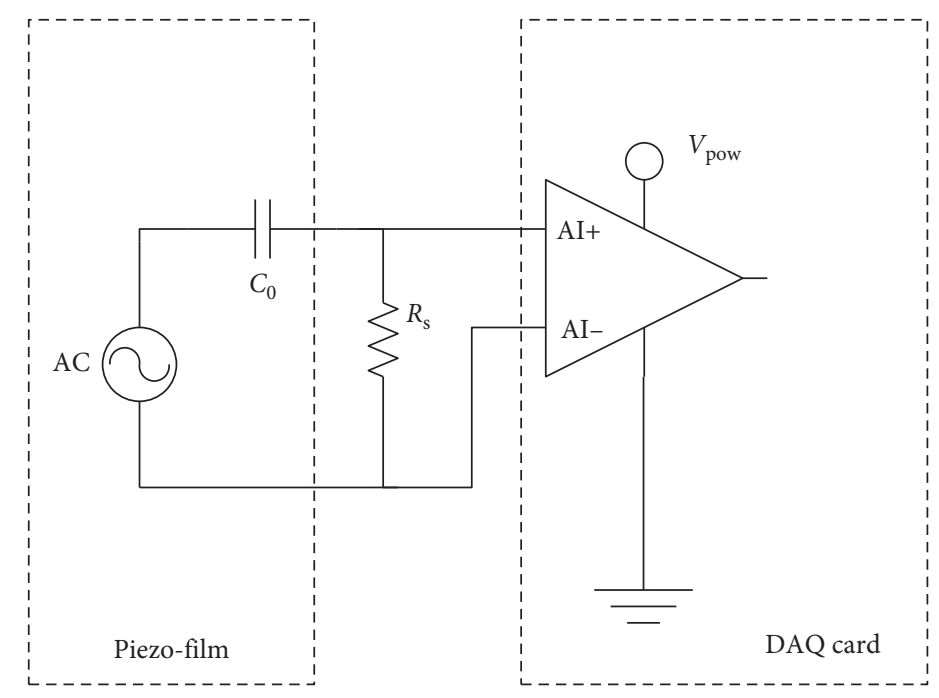

FIgURE 3: Equivalent circuit of piezo-sensor with DAQ card.

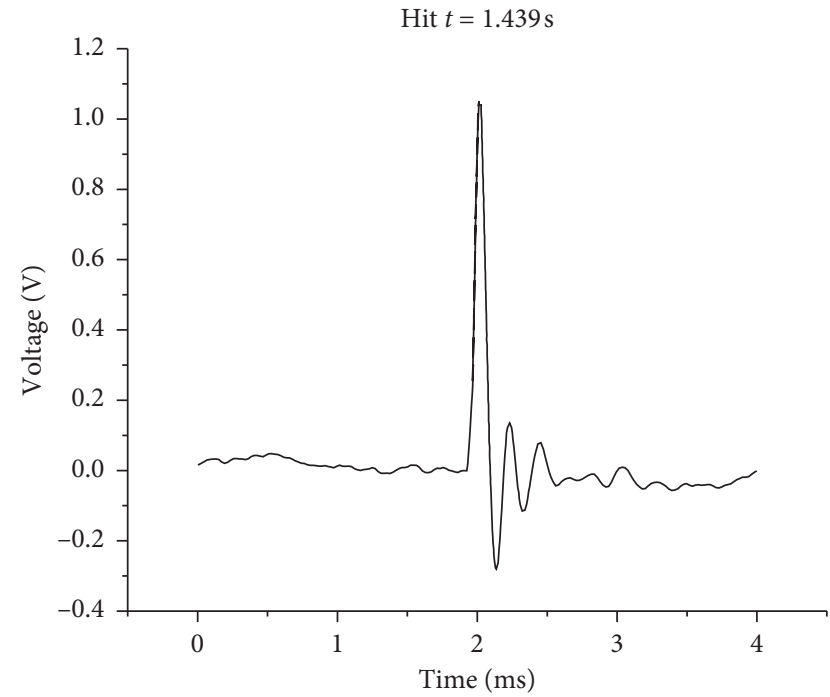

FIgURE 4: Single hit of barley.

3.2. Signal Processing Algorithm. In order to calculate the amount of the seeds, the authors developed and tested a custom algorithm. Its operation is based on the analysis of signal variability. Its parameters were selected on the basis of recorded single beats. The algorithm uses statistics in two adjacent time windows of different lengths. The principle of operation consists of a determination of the standard deviation of the signal in the first window and then comparing it with the standard deviation in the next window. Organigramme of the algorithm is presented in Figure 8 .

The algorithm allows for the detection of impacts on the basics of their difference from the noise. The algorithm is suitable for continuous operation. The windows in which the statistics are taken are moved by a certain fixed vector. The parameters of the developed algorithm are the threshold of triggering (ratio of standard deviations of the signal in both

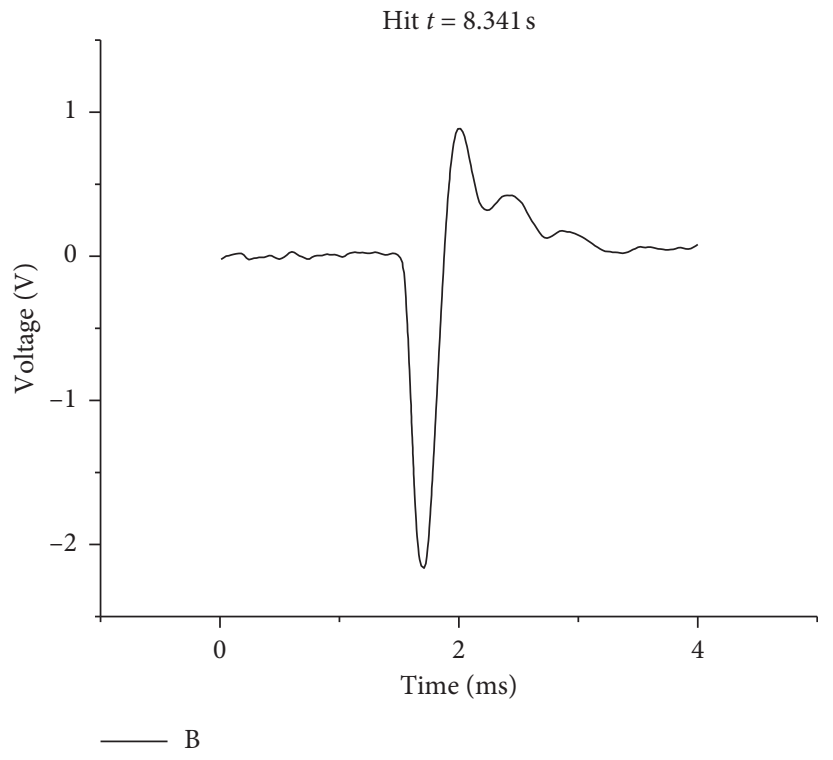

Figure 5: Single hit of barley.

windows) and the length of individual windows. Figure 9 shows an example of an impact detected by means of the developed algorithm together with the marked windows in which the measurement was conducted.

The detection mechanism has a built-in saturation mechanism that enables the avoidance of double counting of strokes that originate from ricketted seeds. The key issue is to choose the right length of window duration. Experimentally, the length of the first window was chosen to be $10 \mathrm{~ms}$, which reflects the balance between the objective averaging of signal power and the value of interference. The width of the second window was set at 2 ms based on the analysis of individual recorded hits. Additionally, in order to avoid interference of noise components with periods comparable to the width of windows, the signal 


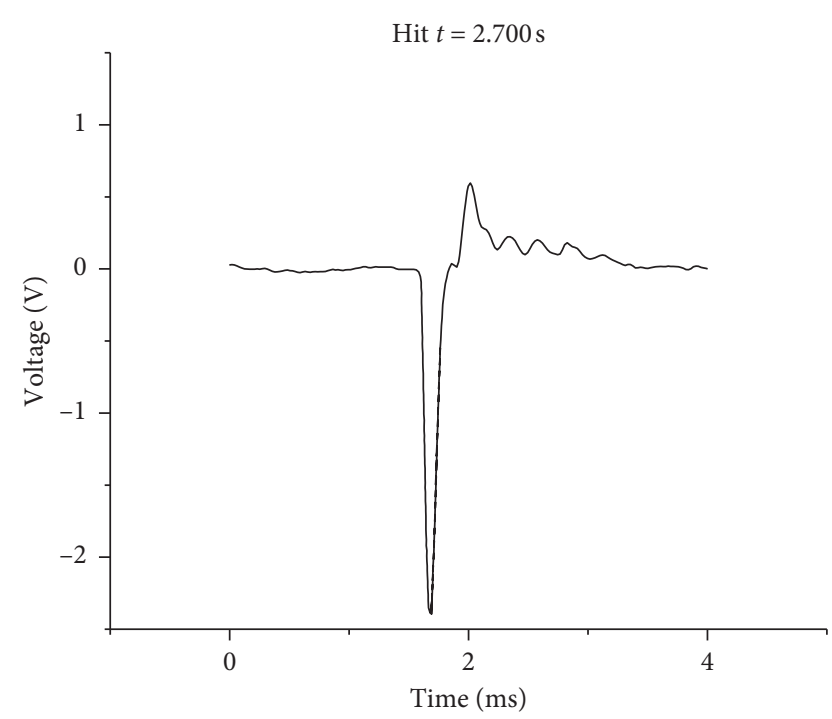

FIgURE 6: Single hit of triticale.

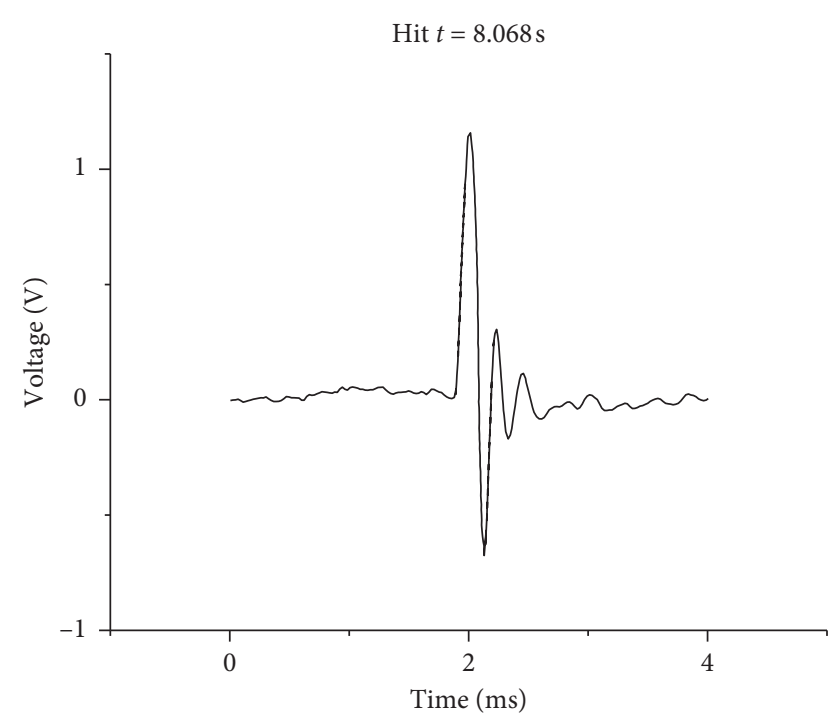

Figure 7: Single hit of triticale.

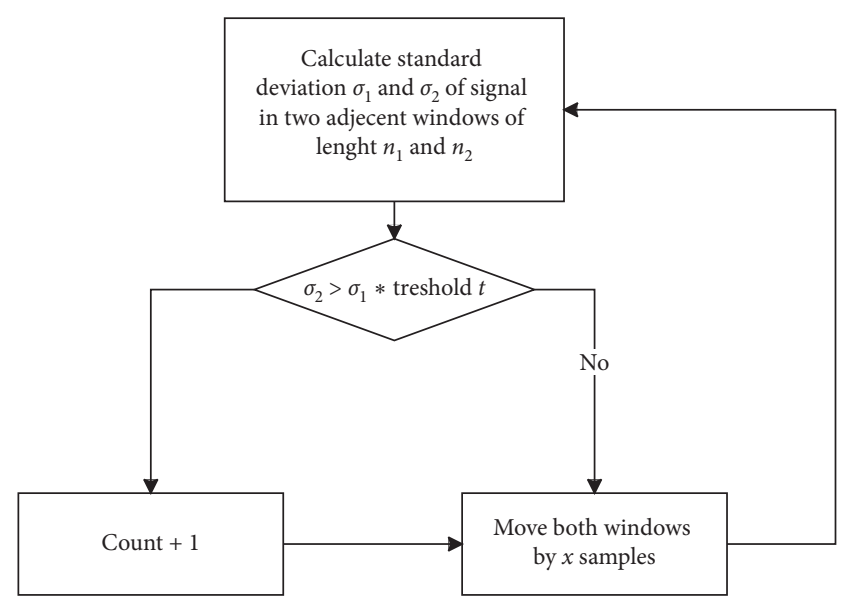

FIGURE 8: Organigramme of the applied algorithm.

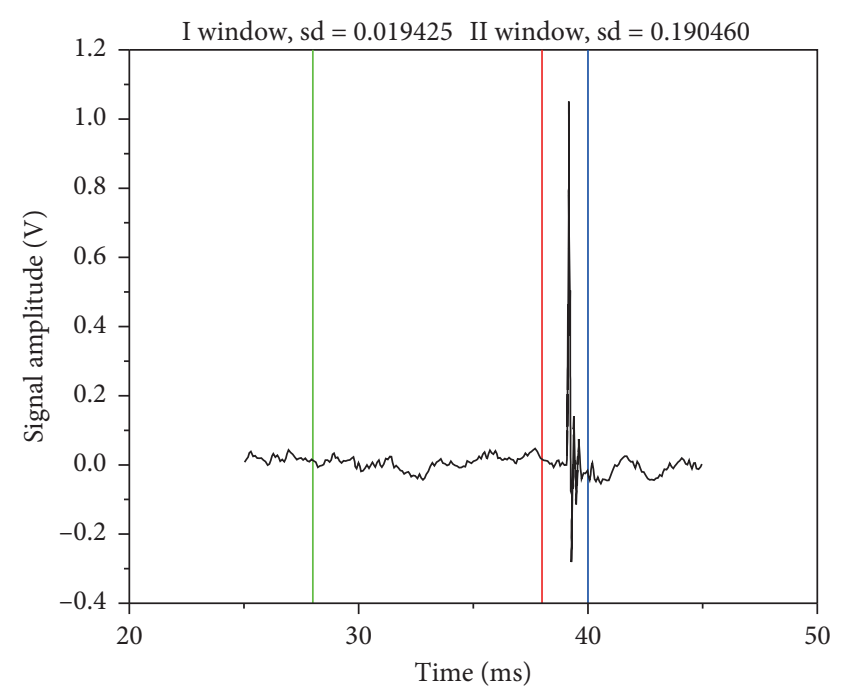

Figure 9: Signal excerpt with two adjacent windows marked. Two windows capture the moment of detection in real time. The window on the left following the window on the right observes the regular signal with the noise, while in the right window it is possible to observe an impact of the type shown in Figure 4. Above both windows, SD values for both windows are presented.

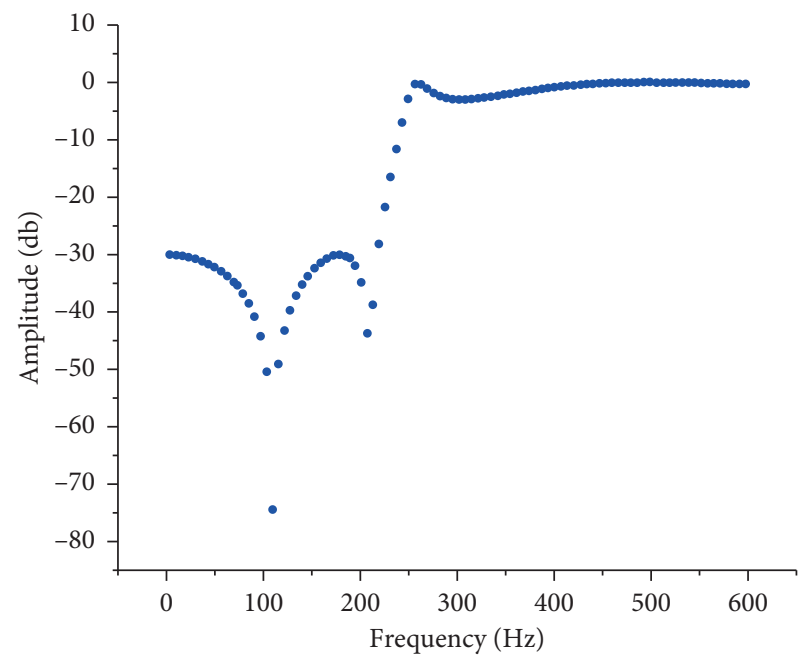

FIgURE 10: The spectrum of high-pass filter applied to recorded signal. The cutoff area lies between 200 and $300 \mathrm{~Hz}$. The filter is characterized by two lobes in the area below $200 \mathrm{~Hz}$, and the flatness of the filtering over $300 \mathrm{~Hz}$ is acceptable.

was filtered by means of an elliptical high-pass filter. The filter order is four, and the parameters selected provide attenuation stronger than $30 \mathrm{db}$ with losses in passband lower than $3 \mathrm{db}$. The transition between the pass-through and blocking band is between 200 and $250 \mathrm{~Hz}$. Figure 10 presents the characteristics of the filter.

The filter corner frequency corresponds to the length of the window used for impact detection, thus removing the effect of periodic noise twice the width of the window that could artificially inflate the standard deviation calculated in the first window. 


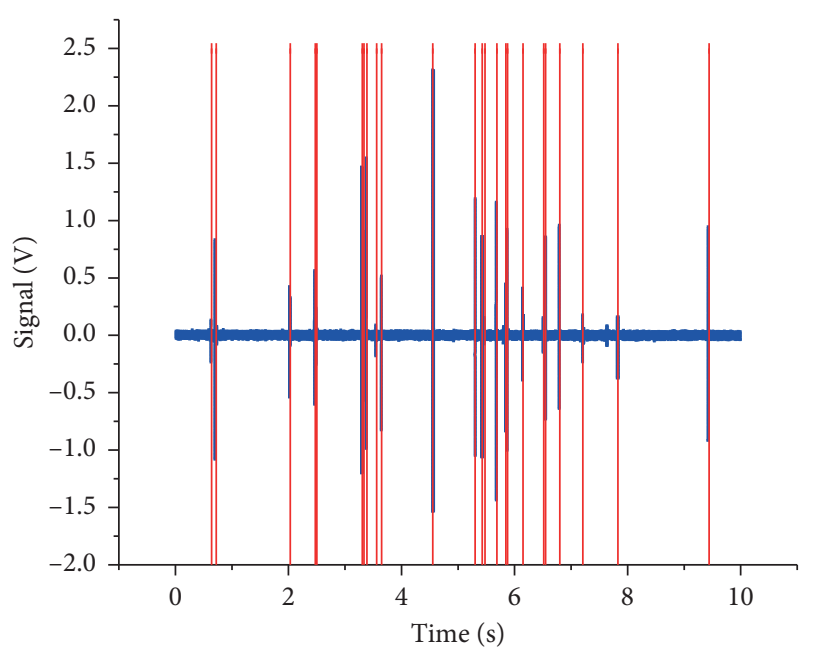

FIgURE 11: Signal with detected peaks marked, threshold $=2.5$. Barley, 50 seeds delivered in batch. Blue lines show signal values, and the red lines denote the detection performed by the algorithm. It is possible to conclude that all significant events are counted, apart from one probable hit between 7.5- $8 \mathrm{~s}$. The figure proves the algorithm's capability to distinguish recoils from original hits for more accurate counting.

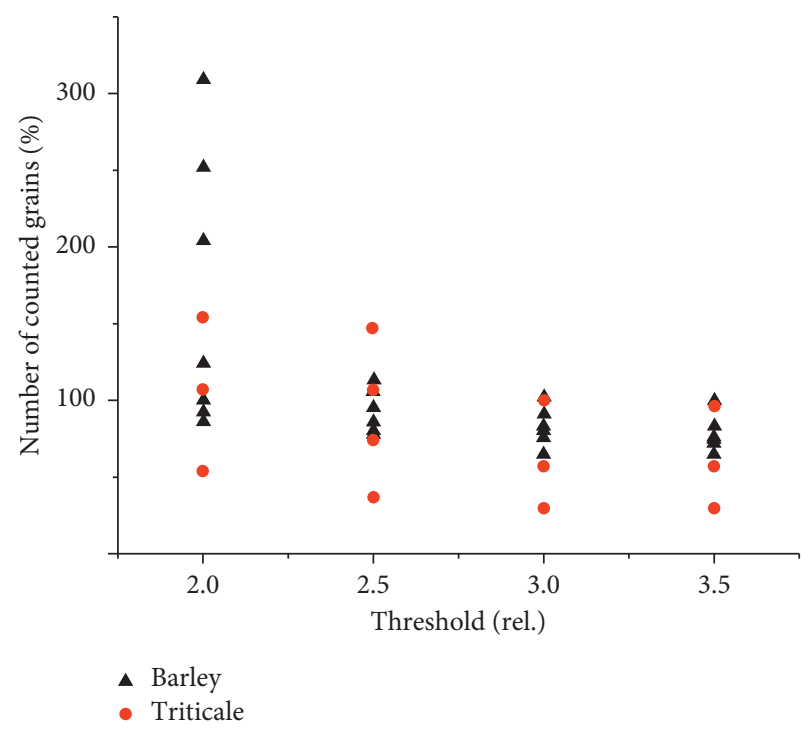

FIGURE 12: Distribution of algorithm results based on repeated experiments using both barley and Triticale versus threshold values. With threshold values below 2.5 the number of false counts can be as high as $\mathbf{3 0 0 \%}$. Similarly, when the threshold is over 3, numerous hits are undercounted.

3.3. Performance Test. The quality test of the counting algorithm was performed on two types of samples. In the first type of sample the seed was dosed by means of a rotary feeder with 23 compartments. The rotation of the feeder lasted about 10 seconds. By placing the seed in individual partitions, it was possible to control its feeding (single or in a group of two or three seeds), about every $450 \mathrm{~ms}$. The second type of recorded signal was a signal generated by seeds thrown in a handful of 50 seeds into a funnel. Knowledge of the number of seeds thrown into the hopper allows for an accurate benchmark of the seeds counting algorithm. Figure 11 is an example of a signal segment with detected hits marked.

It can be seen that the algorithm allows for accurate counting of grain strokes. Figure 12 shows the results for different grain types depending on the different trigger threshold values.

The analysis of the above figure suggests that optimal trigger threshold values were in the range 2.5-3. If the values were lower, there was an effect of incorrect noise counting. Larger values ignored the beats that generated a weak signal, which results in seeds not being counted properly.

\section{Conclusions}

The above work presents a system for counting the seed fed in the seed drill coulter. It consists of a hardware part (measuring card, conditioning system, and piezoelectric sensor) and an efficient signal processing algorithm. The operation of the algorithm and its selected parameters are described. The optimal trigger threshold value is 2.5 for windows with a length of 5 and $2 \mathrm{~ms}$.

For such parameters, the system was able to recognize between $37 \%$ and $113 \%$ of barley and triticale grains, with $80 \%$ of the measurements in a range between $78 \%$ and $113 \%$. This shows that the system could be used in practice, as well as the need for further research. In subsequent iterations to improve the signal quality, it is planned to add charge amplifiers to the signal generated by the piezoelectric sensor. Adding such an amplifier would allow for more accurate impact detection and improve the frequency characteristics of the system [24]. The key to the proper operation of the system is the selection of a trigger threshold, which in the future may require calibration in the real seed drill system. The developed method is promising in terms of implementation into market practice and allows for a significant increase in the quality of seeding and improved yields of the seeding process. It has a number of advantages over classical methods of seed counting in the seed drill, which are based mainly on optical methods. The first advantage is the potential increase in grain counting accuracy due to better separation of light contaminants from actual grains. Both fractions have similar sizes, and thus the optical system will tend to false count contaminations. On the contrary, the system using a piezoelectric sensor, described in the paper, is sensitive to weight and speed differences between contaminants and grains. An additional limitation of the optical system is the need for frequent maintenance due to the accumulation of dust on the lenses. In the proposed sensor, because the grains hit the soft element that covers the piezoelectric sensor, the dustiness of the environment and contamination of the sensor will change the characteristics of its operation to a lesser extent. The promising results of the research allowed for the start of preliminary talks with seeder manufacturers in order to apply the system into industry tests. 


\section{Data Availability}

The measured signal and algorithm data used to support the findings of this study have been deposited in the OSF repository (Paszkiewicz, B. (2019, November 6). PVDF piezoelectric sensors for seeds counting. Retrieved from osf.io/ $7 \mathrm{dkmv})$.

\section{Conflicts of Interest}

There are no conflicts of interest regarding the research presented in this paper.

\section{Acknowledgments}

This work was cofinanced by the National Centre for Research and Development under grants: LIDER VIII project no. LIDER/24/0137/L-8/16/NCBIR/2017 and TECHMATSTRATEG no. 1/346922/4/NCBR/20177, Polish National Agency for Academic Exchange under the contract PPN/ BIL/2018/1/00137, and Wroclaw University of Science and Technology subsidy. This work was accomplished thanks to the product indicators and result indicators achieved within the projects cofinanced by the European Union within the European Regional Development Fund, through a grant from the Innovative Economy (no. POIG.01.01.02-00-008/ 08-05) and by the National Centre for Research and Development through the Applied Research Program Grant no. 178782 and Grant LIDER no. 027/533/L-5/13/NCBR/2014.

\section{References}

[1] Ł. Gierz, "Comparative studies of grain flow sensor in row drills and single seeders," Journal of Research and Applications in Agricultural Engineering, vol. 60, no. 1, 2015.

[2] V. L. Hecht, V. M. Temperton, K. A. Nagel, U. Rascher, and J. A. Postma, "Sowing density: a neglected factor fundamentally affecting root distribution and biomass allocation of field grown spring barley (Hordeum vulgare L.)," Frontiers in Plant Science, vol. 7, p. 944, 2016.

[3] W. S. Lee, V. Alchanatis, C. Yang, M. Hirafuji, D. Moshou, and C. Li, "Sensing technologies for precision specialty crop production," Computers and Electronics in Agriculture, vol. 74, no. 1, pp. 2-33, 2010.

[4] S. Malhotra, S. Bhosale, P. Joshi, and B. Karthikeyan, "Blockage detection in seeder," in Proceedings of the 2016 World Conference on Futuristic Trends in Research and Innovation for Social Welfare (Startup Conclave), pp. 1-4, Coimbatore, India, February 2016.

[5] G. M. Sharipov, D. S. Paraforos, and H. W. Griepentrog, "Modelling and simulation of the dynamic performance of a notill seeding assembly with a semi-active damper," Computers and Electronics in Agriculture, vol. 139, pp. 187-197, 2017.

[6] A. Yatskul, J.-P. Lemiere, and F. Cointault, "Influence of the divider head functioning conditions and geometry on the seed's distribution accuracy of the air-seeder," Biosystems Engineering, vol. 161, pp. 120-134, 2017.

[7] H. J. Heege, "Seeding methods performance for cereals, rape, and beans," Transactions of the ASAE, vol. 36, no. 3, pp. 653-661, 1993.

[8] D. R. Kemp, B. A. Auld, and R. W. Medd, "Does optimizing plant arrangements reduce interference or improve the utilization of space?" Agricultural Systems, vol. 12, no. 1, pp. 31-36, 1983.

[9] Y. Lan, M. F. Kocher, and J. A. Smith, "Opto-electronic sensor system for laboratory measurement of planter seed spacing with small seeds," Journal of Agricultural Engineering Research, vol. 72, no. 2, pp. 119-127, 1999.

[10] Q. X. Chen and P. A. Payne, "Industrial applications of piezoelectric polymer transducers," Measurement Science and Technology, vol. 6, no. 3, pp. 249-267, 1995.

[11] L. Seminara, M. Capurro, P. Cirillo, G. Cannata, and M. Valle, "Electromechanical characterization of piezoelectric PVDF polymer films for tactile sensors in robotics applications," Sensors and Actuators A: Physical, vol. 169, no. 1, pp. 49-58, 2011.

[12] D. Huang, H. Jia, Y. Qi, L. Zhu, and H. Li, “Seeding monitor system for planter based on polyvinylidence fluoride piezoelectric film," Nongye Gongcheng Xuebao/Transactions of the Chinese Society of Agricultural Engineering, vol. 29, no. 23, pp. 15-22, 2013.

[13] X. Jin, K. Zhao, J. Ji, Z. Qiu, Z. He, and H. Ma, "Design and experiment of intelligent monitoring system for vegetable fertilizing and sowing," The Journal of Supercomputing, vol. 76, no. 5, 2020.

[14] Mueller-Elektronik, AIRidium ${ }^{\circledR}$ System, 2019, https:// www.mueller-elektronik.de/en/products/airi.

[15] M. O. Binelo, R. F. De Lima, O. A. Khatchatourian, and J. Stránský, "Modelling of the drag force of agricultural seeds applied to the discrete element method," Biosystems Engineering, vol. 178, pp. 168-175, 2019.

[16] D. Karayel, M. Wiesehoff, A. Ö Eseho, and J. Müller, "Laboratory measurement of seed drill seed spacing and velocity of fall of seeds using high-speed camera system," Computers and Electronics in Agriculture, vol. 50, no. 2, pp. 89-96, 2006.

[17] Ł. Gierz, Z. Staszak, D. Wojcieszak, and K. Koszela, "The validation of the method of speed test of seeds moving in a tube of a pneumatic seed drill," in Proceedings of the SPIE-The International Society for Optical Engineering, Munich, Germany, June 2019.

[18] T. Szymański, M. Wośko, B. Paszkiewicz, B. Paszkiewicz, R. Paszkiewicz, and I. Sankowska, "Growth and coalescence control of inclined c-axis polar and semipolar GaN multilayer structures grown on Si (111), Si (112), and Si (115) by metalorganic vapor phase epitaxy," Journal of Vacuum Science \& Technology A: Vacuum, Surfaces, and Films, vol. 34, no. 5, 2016.

[19] A. Yatskul and J.-P. Lemiere, "Establishing the conveying parameters required for the air-seeders," Biosystems Engineering, vol. 166, pp. 1-12, 2018.

[20] A. Bursztynowicz, Laboratory Analysis of Pneumatic MultiCoulter Seed Drill Control System, Poznan University of Technology, Poznań, Poland, 2015.

[21] Z. Zhao, Y. Li, J. Chen, and J. Xu, "Grain separation loss monitoring system in combine harvester," Computers and Electronics in Agriculture, vol. 76, no. 2, pp. 183-188, 2011.

[22] P. Ueberschlag, "PVDF piezoelectric polymer," Sensor Review, vol. 21, no. 2, pp. 118-126, 2001.

[23] L. F. Brown and D. L. Carlson, "Ultrasound transducer models for piezoelectric polymer films," IEEE Transactions on Ultrasonics, Ferroelectrics and Frequency Control, vol. 36, no. 3, pp. 313-318, 1989.

[24] I. E. Tothill, "Biosensors developments and potential applications in the agricultural diagnosis sector," Computers and Electronics in Agriculture, vol. 30, no. 1-3, pp. 205-218, 2001. 